\section{phospholipase C gamma-1 is required downstream of vascular endothelial growth factor during arterial development}

Nathan D. Lawson, ${ }^{1,2}$ Joshua W. Mugford, Brigid A. Diamond, and Brant M. Weinstein

Laboratory of Molecular Genetics, National Institute of Child Health and Human Development, National Institutes of Health, Bethesda, Maryland 20892, USA

In this study, we utilize transgenic zebrafish with fluorescently labeled blood vessels to identify and characterize a mutant (y10) that displays specific defects in the formation of arteries, but not veins. We find that y10 encodes phospholipase $C$ gamma-1 (plcg1), a known effector of receptor tyrosine kinase signaling. We further show that $\operatorname{plcg}^{\text {y10 }}$ mutant embryos fail to respond to exogenous Vegf. Our results indicate that Plcg1 functions specifically downstream of the Vegf receptor during embryonic development to govern formation of the arterial system.

Received January 3, 2003; revised version accepted April 14, 2003.

Historically, the structural differences between arteries and veins were thought to be the result of physiological factors such as the pressure and direction of blood flow (Lawson and Weinstein 2002b). However, recent work demonstrates that a number of signaling pathways specifically induce the differentiation of arterial endothelial cells before circulation commences (Lawson et al. 2001, 2002; Mukouyama et al. 2002; Visconti et al. 2002). In particular, vascular endothelial growth factor (Vegf) is required for the development of arteries (Mukouyama et al. 2002; Stalmans et al. 2002) and the differentiation of arterial endothelial cells (Lawson et al. 2002; Mukouyama et al. 2002) during embryonic development in both zebrafish and mouse. Despite the wealth of information concerning components of the Vegf signaling pathway in endothelial cell lines (Zachary and Gliki 2001), little is known about the downstream factors that are necessary in vivo during blood vessel formation and arterial development. In this study, we have taken advantage of transgenic zebrafish with fluorescently labeled blood vessels (Lawson and Weinstein 2002a) in conjunction with a forward genetic screen to identify a gene within the Vegf signaling pathway that is required for arterial development. We find that y10 mutant ze-

[Keywords: zebrafish; endothelial; artery; Plcg1; Vegf]

${ }^{1}$ Present address: Program in Gene Function and Expression, University of Massachusetts Medical School, Worcester, MA 01605.

${ }^{2}$ Corresponding author.

E-MAIL Nathan.Lawson@umassmed.edu; FAX (508) 856-5460.

Article and publication are at http://www.genesdev.org/cgi/doi/10.1101/ gad.1072203. brafish embryos display defects in the formation of arteries, but not veins, and are deficient in the expression of artery-specific markers such as ephrin-B2a, similar to zebrafish and mouse embryos lacking Vegf function (Lawson et al. 2002; Mukouyama et al. 2002; Stalmans et al. 2002). We show that $y 10$ encodes the zebrafish homolog of phospholipase C gamma-1 (plcg1) a known downstream effector of numerous receptor tyrosine kinases (Rhee 2001). Using $p \operatorname{lcg} 1^{y 10}$ mutant embryos together with microinjection of vegf mRNA, we are able to demonstrate that Plcgl is required for normal Vegf function. Taken together, our observations reveal a specific requirement for Plcg1 during formation and differentiation of arteries, and indicate that Vegf is the primary growth factor that utilizes Plcg1 signaling during embryonic development.

\section{Results and Discussion}

To identify putative regulators of Vegf signaling, we performed a screen for mutants lacking segmental blood vessels (N.D. Lawson, J.W. Mugford, B.A. Diamond, and B.M. Weinstein, unpubl.), a phenotype associated with loss of Vegf function in zebrafish embryos (Nasevicius et al. 2000). As a screening tool, we utilized zebrafish harboring a fli1:enhanced green fluorescent protein transgene [TG(fli1:egfp $)^{y 1}$; Lawson and Weinstein 2002a] to enable fluorescent visualization of developing blood vessels in vivo. Using this strategy, we identified the zebrafish y10 mutant. In both wild-type and homozygous y10 mutant embryos, overall morphology is normal at 30 $\mathrm{h}$ post fertilization (hpf; Fig. 1a,d). The somites and notochord, both of which are required for normal development of the trunk blood vessels (Brown et al. 2000; Parsons et al. 2002), appear normal by light microscopy (data not shown). Primary segmental vessels, which sprout from the dorsal aorta, are formed by $30 \mathrm{hpf}$ in wild-type TG(fli1:egfp $)^{y^{1}}$ sibling embryos (Fig. 1b). These vessels are absent in TG(fli1:egfp $)^{y 1}$ embryos mutant for y10 (Fig. 1e). This phenotype is not a general defect in sprouting blood vessel growth, or angiogenesis, as secondary segmental vessels, which sprout from the posterior cardinal vein by $50 \mathrm{hpf}$ and contribute to parachordal vessels in wild-type embryos (Fig. 1c), are apparent in y10 mutant embryos (Fig. 1f). The midcerebral vein, a blood vessel that forms adjacent to the midbrain-hindbrain boundary via angiogenesis, also forms normally in y10 mutant embryos (Fig. 1g,h). In zebrafish embryos, the lateral dorsal aortae connect the outflow tract of the heart to the trunk circulatory network and fuse in the anterior trunk to give rise to a single dorsal aorta (Isogai et al. 2001). In wild-type embryos, the lateral dorsal aortae are lumenized by $30 \mathrm{hpf}$ (Fig. 1i), whereas in y10 mutant embryos, these blood vessels appear thin and fail to lumenize (Fig. 1j). In contrast, the branches of the posterior cardinal vein are normal in mutant embryos (Fig. 1, cf. i and j). These observations indicate that y10 mutant embryos display a specific defect in the formation of arteries, whereas the development of veins appears unaffected. Consistent with these defects in blood vessel morphology, y10 mutant embryos fail to exhibit head or trunk circulation and have severe pericardial edema by $2 \mathrm{~d}$ postfertilization $(\mathrm{dpf}$; data not shown). 


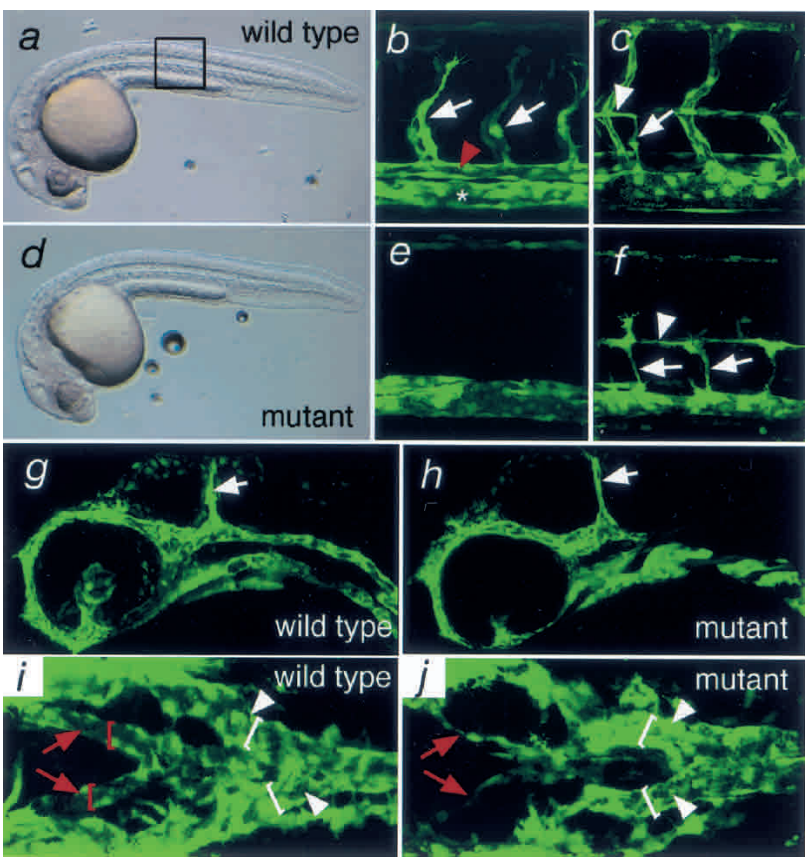

Figure 1. Vascular morphology in $T G(f l i 1: e g f p)^{y 1} ; y 10$ wild-type and mutant embryos. (a) Transmitted light image of a wild-type sibling embryo at $30 \mathrm{hpf}$. Boxed region indicates views in $b$ and $c$ (wild type) and $e$ and $f$ (mutant). (b) Primary segmental vessels (white arrows) sprout from the dorsal aorta (red arrowhead) in a wild-type embryo at $30 \mathrm{hpf}_{\text {; }}$ the posterior cardinal vein is indicated with an asterisk. (c) A secondary segmental vessel (white arrow) sprouts from the posterior cardinal vein and contributes to the parachordal vessel (white arrowhead) at $50 \mathrm{hpf}$ in a wild-type embryo. (d) Transmitted light image of a y10 mutant embryo at $30 \mathrm{hpf}$. (e) Primary segmental vessels fail to form in y10 mutant embryos; $30 \mathrm{hpf}$ time point. $(f)$ Secondary segmental vessels (white arrows) sprout from the posterior cardinal vein and give rise to the parachordal vessel (white arrowhead) in y10 mutant embryos at $50 \mathrm{hpf}$ ( $(\mathrm{g})$ Midcerebral vein (white arrow) in a wild-type embryo at $30 \mathrm{hpf}$. (h) The midcerebral vein (white arrow) forms normally in $30 \mathrm{hpf}$ y10 mutant embryos. (i) Dorsal view of the lateral dorsal aortae (red arrows) and branches of posterior cardinal vein in a wild-type embryo at $30 \mathrm{hpf}$; lumens of the lateral dorsal aortae (red brackets) and cardinal vein branches (white brackets) are indicated. (j) Dorsal view of lateral dorsal aortae in y10 mutant embryos. Note the poor lumenization and formation of lateral aortae branches (red arrows) compared with the posterior cardinal vein (white arrowheads; lumens indicated by white brackets). $(a-h)$ Lateral views; anterior is to the left, dorsal is at top. Images in $b, c, e$, and $f$ were obtained by multiphoton laser scanning microscopy, whereas $g-j$ were obtained using a confocal laser microscope. $(g, h)$ Optical sections were limited to those containing the midcerebral vein to minimize flourescence derived from the aortic arch mesenchyme.

In addition to defects in artery formation, y10 mutant embryos exhibit a loss of artery-specific gene expression in the dorsal aorta. By 24 hpf, wild-type embryos express ephrin-B2a in the dorsal aorta (Fig. 2a), whereas in approximately half of y10 mutant embryos, ephrin-B2a expression is strongly reduced or absent (Figs. $2 \mathrm{~b}, 4 \mathrm{~g}$, below). Dorsal aorta-specific expression of notch5 (Fig. 2c), an important regulator of arterial differentiation downstream of Vegf (Lawson et al. 2001, 2002), is also reduced or absent at a frequency similar to ephrin-B2a (Fig. 2d; data not shown). We find that general endothelial cell markers, such as $f 1 k 1$ and tie 1 are expressed in the major trunk blood vessels, and their expression appears to be mildly reduced in y10 mutant embryos (Fig. 2e-h). Ex- pression level of fli1 transcript (Thompson et al. 1998) in the dorsal aorta and posterior cardinal vein does not appear to be affected in y10 mutant embryos (Fig. 2i,j), consistent with the equivalent level of fli1:egfp transgene expression in wild-type and mutant embryos (Fig. 1). The semipenetrant nature of the loss in artery marker gene expression is consistent with our previous observations that numerous pathways likely contribute to the differentiation of arterial endothelial cells in the zebrafish dorsal aorta during embryogenesis (Lawson et al. 2002). In addition, the appearance of endothelial cells in the position of the dorsal aorta indicates a failure of these cells to undergo arterial differentiation rather than their inability to migrate to the correct location.

The phenotype of y10 mutant embryos is similar to zebrafish or mouse embryos lacking Vegf function (Lawson et al. 2002; Mukouyama et al. 2002; Stalmans et al. 2002). However, y10 does not map to vegf or its receptor, flk1 (data not shown). To identify the gene responsible for the y10 mutant phenotype, we performed bulk segregant analysis on wild-type and mutant embryos and mapped y10 to within $0.9 \mathrm{cM}$ of Z6376 on linkage group 23 (Fig. 3a). By comparing the available zebrafish mei-

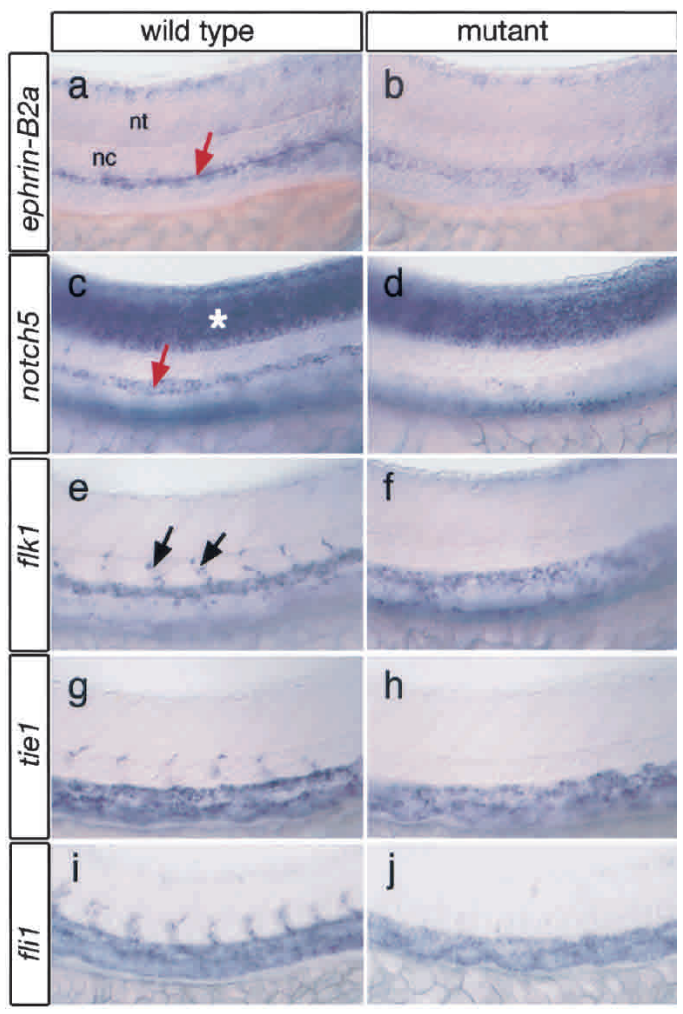

Figure 2. Loss of artery marker gene expression in y10 mutant embryos. (a) Normal ephrin-B2a in dorsal aorta (red arrow) of a wildtype sibling embryo. nt, neural tube; nc, notochord. (b) y10 mutant embryo with reduced dorsal aorta ephrin-B2a expression. (c) notch5 expression in dorsal aorta (red arrow) and neural tube (asterisk) of wild-type sibling embryo. (d) Reduced dorsal aorta notch5 expression in y10 mutant embryo. Neural tube expression in not affected. (e) flk1 expression in wild-type sibling embryo. Arrows indicate segmental vessels. $(f)$ flk 1 expression in a y10 mutant embryo. $(g)$ tie 1 expression in a wild-type sibling embryo. (h) tie 1 expression in a y10 mutant embryo. (i) fli1 expression in a wild-type embryo. (j) fli1 expression in a y10 mutant embryo. Note absence of segmental vessels in $f, h$, and $j .(a-f)$ Lateral views of 24 hpf zebrafish embryo trunk; anterior is to the left, dorsal is up. 
a
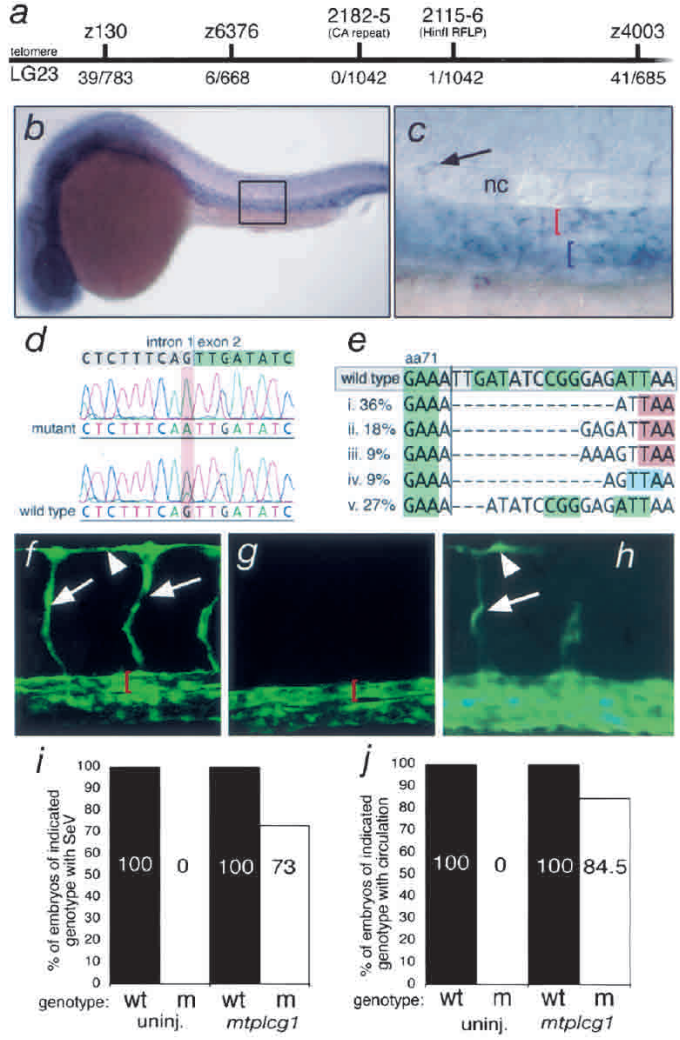
(herentalion or segmental vessels (SeV) at 30-32 hpf, and then genotyped using oligonucleotide primers 2182 and 2185. (h) Epifluorescence micrograph of a TG(fli1:egfp $)^{\text {y1 }}$ embryo mutant for plcg $1^{\text {y10 }}$ with partial rescue of segmental vessel (arrow) and dorsal logitudinal anastomotic vessel (arrowhead) formation. (i) MTplcg1 rescues SeV formation in plcg1 10 mutant embryos. Results represent average of two experiments. Partial SeV formation was scored as rescue. (j) MTplcg1 rescues circulation in plcg $1^{y 10}$ mutant embryos. Results represent the average from three experiments.

otic, radiation hybrid, and heat-shock maps (http://zfin. org/cgi-bin/webdriver?MIval=aa-mapperselect.apg), we found that several expressed sequence tags (EST) with homology to rat plcg1 were located in this region (data not shown). Because Plcg1 activity can be induced through the Vegf receptor (Takahashi et al. 2001) and the artery defect in y10 mutant embryos is reminiscent of loss of Vegf phenotypes in zebrafish (Lawson et al. 2002) and mouse (Mukouyama et al. 2002; Stalmans et al. 2002) embryos, plcg1 represented a viable candidate gene for y10. Mapping of polymorphic markers in the plcg1 gene indicated that it is linked tightly to y10 (Fig. 3a). In addition, plcg1 mRNA is expressed in the vasculature of zebrafish embryos at $24 \mathrm{hpf}$ (Fig. 3b,c) in both the dorsal aorta and posterior cardinal vein, as well as in segmental vessel sprouts (Fig. 3c).

Sequence analysis of the plcg1 gene from wild-type and mutant embryos revealed that a consensus splice acceptor site at the intron 1-exon 2 boundary of plcg1 is eliminated by a G-to-A transition in mutants (Fig. 3d) and is associated with variable size deletions in the $p l c g 1$ transcript (Fig. 3e). The majority of these deletions result in a frame shift into a premature stop codon and truncate the Plcg1 protein at amino acid 73 (Fig. 3e). Less than $30 \%$ of the transcripts lack only three nucleotides and result in loss of $\mathrm{I}_{72}$ and change of $\mathrm{D}_{73}$ to $\mathrm{N}$. Both of these amino acids are identical in rat, human, and zebrafish Plcg1 (data not shown) and are located in the pleckstrin homology domain, which is important for growth factor-
Figure 3. $y 10$ is a mutation in phospholipase C gamma-1. (a) Telomeric region of zebrafish linkage group 23. Polymorphic markers used in this study are shown above the map, whereas numbers below the map indicate number of recombinants per informative meioses. Z130, Z6376, and Z4003 are CA repeats from the initial bulk segregant mapping panel. 2115-2116 encompasses sequence within the plcg13'UTR that includes a HinfI restriction fragment length polymorphism (RFLP). 2182-2185 is a CA-repeat flanking the exon that encodes Plcg1 amino acids 337-365. $(b, c)$ Whole-mount in situ hybridization using a plcg1 riboprobe at $24 \mathrm{hpf}$. Lateral views; dorsal is up. (b) plcg1 is expressed in the trunk blood vessels and diffusely in the head; boxed region indicates area magnified in $c .(c)$ plcg1 expression in the dorsal aorta (red bracket) and posterior cardinal vein (blue bracket) and segmental vessel (arrow); the embryo in this image is slanted to allow simultaneous visualization of segmental vessels and the major trunk vessels. (d) Gray and green highlighted sequences indicate consensus genomic trace sequences for plcg1 intron 1 and exon 2, respectively. A G $\rightarrow$ A transition in the conserved AG splice acceptor sequence is highlighted in red. $(e)$ Variable size deletions found within the $\operatorname{plcg} 1$ coding sequence in cDNA derived from y10 mutant embryos. Numbers on the left indicate the frequency of particular transcripts $(n=11)$. Green boxes denote alternating codons of wild-type plcg1 coding sequence. Red boxes indicate premature stop codons present in transcripts i-iii. Light blue box in transcript iv indicates a frameshift; this transcript encodes an additional 53 amino acids before terminating in a premature stop codon. Black vertical line indicates position of exon 1-exon 2 splice junction. $(f, g)$ Confocal laser microscope images; lateral views of the trunk; anterior is to the left and dorsal is up. Red brackets indicate lumen of the dorsal aorta. (f) Wild-type $T G(f l i 1: e g f p)^{y 1}$ embryo injected with $15 \mathrm{ng}$ of a control scrambled morpholino that displays fully formed segmental vessels (arrows) and dorsal longitudinal anastomotic vessel (arrowhead). (g) TG(fli1:egfp $)^{y 1}$ embryo injected with $15 \mathrm{ng}$ of plcg1 morpholino with no segmental vessel sprouts. $(h-j)$ Embryos derived from a $p l c g 1^{\text {y10/+ }}$ incross were injected with $100 \mathrm{pg}$ of myc-epitope-tagged plco1 $\mathrm{mt}$

induced membrane localization of Plcg1 (Falasca et al. 1998). To confirm that the plcg1 exon 1-exon 2 junction was affected in $p l c g 1^{y 10}$ mutant embryos, we designed an antisense Morpholino oligonucleotide against the exon 1 -intron 1 boundary sequence to interfere with plcg1 pre-mRNA splicing (Draper et al. 2001). Wild-type TG(fli1:egfp $)^{y 1}$ embryos injected with a scrambled control Morpholino display a fully formed primary segmental blood vessel network by 32 hpf (Fig. 3f; 111 of 111 in 3 experiments) and normal circulation (86 of 89 in 3 experiments; data not shown). In contrast, TG(fli1:egfp $)^{\mathrm{y} 1}$ embryos injected with the plcg1 Morpholino display reduced numbers of segmental vessel sprouts (Fig. $3 g_{;} 52$ of 106 with $<5$ segmental vessels in 3 experiments) and loss of circulation (62 of 83 without circulation in 3 experiments; data not shown). To confirm that loss of plcg1 function was responsible for the vascular defects in y10 mutant embryos, we injected mRNA encoding a myctagged form of Plcg1 into embryos derived from a plcg $1^{\text {y1O}}$ incross. The resulting embryos were then scored for presence or absence of segmental blood vessels or circulation at $30 \mathrm{hpf}$ and subsequently genotyped using the 2182-2185 CA marker. In uninjected embryos, all wild-type siblings display fully formed segmental blood vessels or active circulation by $30 \mathrm{hpf}$, whereas mutants do not (data not shown; Fig. 3i,j). In contrast, we find that $73 \%$ of mutant embryos that were injected with mtplcg1 mRNA form segmental blood vessels (Fig. 3h,i) and $85 \%$ of embryos with a mutant genotype display 
circulation at $30 \mathrm{hpf}$ (Fig. 3j; data not shown). Together, these results establish definitively that the gene responsible for the y10 mutant phenotype encodes the zebrafish homolog of Plcg1.

Biochemical studies have demonstrated that Plcg1 can be an effector of Vegf signaling in endothelial cell lines (Takahashi and Shibuya 1997; Takahashi et al. 2001), and evidence from mice lacking plcg1 indicates that it is required for blood vessel development (Liao et al. 2002). However, there is no definitive genetic evidence to confirm that Plcg1 functions downstream of Vegf in vivo. The phenotype of zebrafish plcg1 $1^{\text {y10 }}$ mutant embryos and the similarity to mice (Mukouyama et al. 2002; Stalmans et al. 2002) or zebrafish (Nasevicius et al. 2000; Lawson et al. 2002) lacking Vegf function, suggests that Plcg1 is an important downstream effector of Vegf during artery development. The availability of $p l c g 1^{y 10}$ mutant zebrafish allowed us to address whether or not this was the case. To determine whether Plcg1 was required for Vegf function, we injected vegf $f_{121}$ mRNA into embryos derived from $p l c g 1^{y 10}$ heterozygous carriers. We then assayed for flk1 or ephrin-B2a expression in injected embryos, followed by PCR analysis to identify wild-type and mutant embryos. We have found previously that exogenous Vegf induces the expression of both of these markers, although induction of ephrin-B2a requires the Notch signaling pathway, whereas induction of $f 1 k 1$ does not (Lawson et al. 2002). Consistent with previous observations (Liang et al. 2001; Lawson et al. 2002), we find that nearly all wild-type embryos injected with $v e g f_{121}$ mRNA display increased levels of flk1 expression in their trunk blood vessels (Fig. 4a,c). In contrast, ectopic Vegf $_{121}$ fails to induce $f l k 1$ expression in $p l c g 1^{\text {y1O }}$ mutant embryos (Fig. 4b,c). Similarly, nearly all wild-type embryos injected with vegf $f_{121}$ mRNA also exhibit ectopic expression of ephrin-B2a in the posterior cardinal vein (Fig. 4f,h). However, plcg $1^{y 10}$ mutant embryos injected with mRNA encoding Vegf $_{121}$ display either normal or reduced ephrin-B2a expression (Fig. 4d,e,h) in proportions similar to uninjected mutant embryos (Fig. 4h). These data indicate that Plcg1 function is required for induction of both Notch-dependent and Notch-independent signaling downstream of Vegf in vivo and is consistent with biochemical evidence that shows Plcg1 functions proximal to the Vegf receptor, Flk1 (Takahashi et al. 2001).

Although Plcg1 is know to function downstream of numerous receptor tyrosine kinases (Rhee 2001; Wilde and Watson 2001), we find that the primary defects in zebrafish $\operatorname{plcg} 1^{\mathrm{y} 10}$ mutants are restricted to the vasculature within the embryo and are remarkably specific to a subset of blood vessels. The similarity of the $p \operatorname{lcg} 1^{\text {y10 }}$ mutant phenotype to zebrafish embryos lacking Vegf (Nasevicius et al. 2000; Lawson et al. 2002) and the failure of $p \operatorname{lcg} 1^{\text {y1O }}$ mutant embryos to respond to exogenous Vegf (this study) indicate that Plcg1 function is required downstream of Vegf to drive arterial development. This requirement for Plcg1 to mediate Vegf signaling during vascular development appears to be conserved in vertebrates, as mice lacking plcg1 also display severe defects in blood vessel formation (Liao et al. 2002) during embryogenesis, similar to those associated with loss of Vegf (Carmeliet et al. 1996). However, mice lacking either Plcg1 or Vegf display a more severe phenotype, including failure to express general endothelial cell markers indicative of an early block in the formation of endothelial
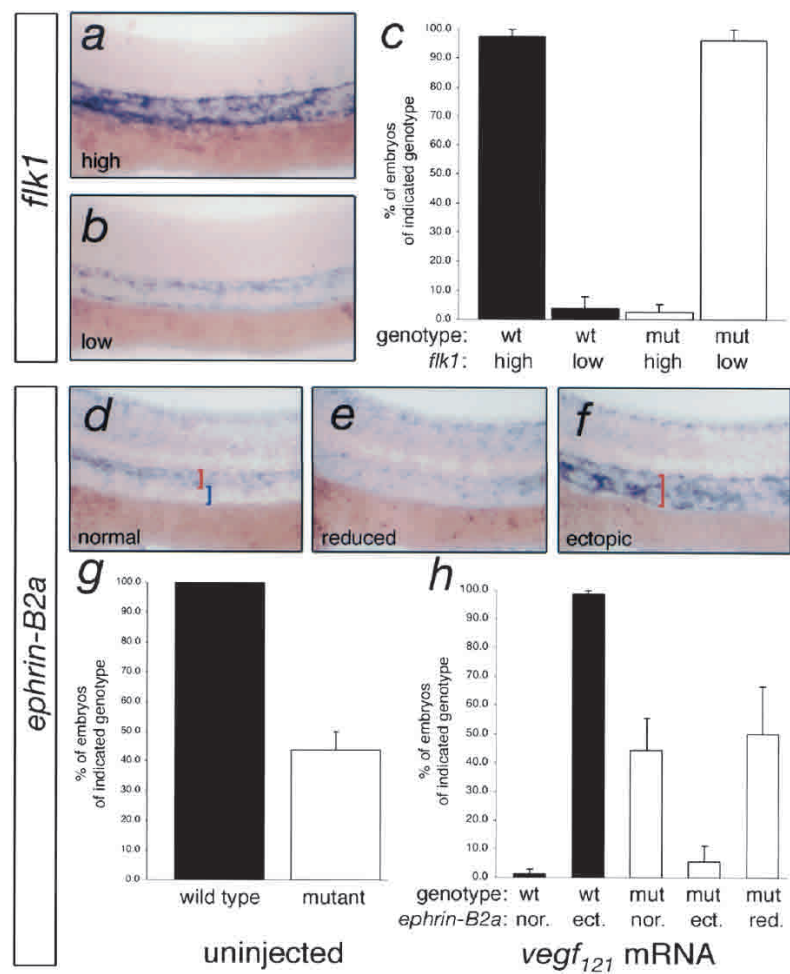

Figure 4. Plcg1 is required for Vegf function. $(a, b)$ Whole-mount in situ hybridization for flk 1 expression in trunk blood vessels of embryos derived from a plcg $1^{+/ y 10}$ incross and injected with $25 \mathrm{pg}$ of $v g f_{121}$ mRNA. Lateral views; anterior is to the right and dorsal is up. (a) Induced (high) expression of $f 1 k 1$. (b) Low flk 1 expression. (c) Chart indicating genotype of embryos expressing high or low levels of $f 1 k 1$. Greater than $90 \%$ of wild-type embryos exhibit induction of flk1 expression, whereas Vegf ${ }_{121}$ fails to induce flk1 expression in mutant embryos. $(d-f)$ ephrin-B2a expression in the trunk blood vessels of embryos derived from $\mathrm{plcg} 1^{+/ y 10}$ carriers injected with 50 pg of $v e g f_{121}$ mRNA. Lateral views; anterior is to the left and dorsal is up. (d) Normal expression of ephrin-B2a in the dorsal aorta (red bracket), but not the posterior cardinal vein (blue bracket) within the trunk. $(e)$ Reduced expression of ephrin-B2a. $(f)$ Ectopic expression of ephrin-B2a expanded into the region of the posterior cardinal vein (red bracket). ( $g$ ) Chart indicating proportion of uninjected embryos displaying ephrin-B2a expression. All uninjected wild-type embryos display normal expression of ephrin-B2a, whereas approximately half of the mutants do not. (h) Proportion of genotyped embryos injected with vegf $f_{121}$ mRNA that display normal, ectopic, or reduced ephrin-B2a expression within the trunk blood vessels. Nearly all wild-type embryos (black bars) injected with vegf $f_{121}$ mRNA show ectopic expression of ephrin-B2a. Less than $10 \%$ of mutant embryos display ectopic expression, whereas the remaining embryos display normal or reduced expression similar to uninjected mutant embryos.

cells from mesodermal precursors, than in zebrafish embryos with comparable loss of function (Nasevicius et al. 2000; Lawson et al. 2002). The reason for these differences is unknown at this time, but may reflect additional redundancy in the Vegf-signaling pathway in zebrafish that allows for early stages of endothelial progenitor cell development. Therefore, only defects in arterial development, which have been observed in mice lacking specific isoforms of Vegf (Stalmans et al. 2002), are observed in the blood vessels of $p l c g 1^{\text {y10 }}$ mutant embryos.

Despite apparent species-specific differences in Vegf sensitivity during embryonic development, recent evi- 
dence indicates that Vegf is an important signal that specifically drives development of the arterial system in both zebrafish and mice (Lawson et al. 2002; Mukouyama et al. 2002; Stalmans et al. 2002; Visconti et al. 2002) and does so through the action of the Notch signaling pathway (Lawson et al. 2001, 2002; Lawson and Weinstein 2002b). However, little is known about the downstream effectors of Vegf that mediate this effect on the developing vasculature in vivo. With the ability to perform screens for mutant blood vessel phenotypes using TG(fli1:egfp $)^{y 1}$ embryos, we are able to demonstrate that the zebrafish is an ideal model to dissect this signaling pathway. The studies presented here validate this forward genetic approach by describing the identification of plcg1 as a necessary component of the arterial differentiation pathway downstream of Vegf. It is likely that identification and characterization of additional mutants with similar phenotypes will yield further insight into the components of the Vegf pathway required for this particular aspect of blood vessel formation.

\section{Materials and methods}

Fish and handling

Zebrafish were maintained and bred as described elsewhere (Westerfield 1993). The TG(fli1:egfp $)^{y 1}$ transgenic line has been described previously (Lawson and Weinstein 2002a).

\section{Mapping}

To facilitate genetic mapping, the TG(fli1:egfp $)^{y 1}$;plcg $1^{y 10}$ founder female was crossed to the wild-type TL line. Identified mutant carriers derived from this map cross were incrossed at sexual maturity, and their embryos were scored on the basis of the presence or absence of circulation at $2 \mathrm{dpf}$. Bulk segregant analaysis was performed using a 192 marker panel of available CA markers. The list of markers in this panel is available upon request. Genomic DNA isolation and PCR, as well as cycle sequencing of PCR products to identify polymorphisms were performed as described elsewhere (Roman et al. 2002). Oligonucleotide sequences for Z130, Z6376, and Z4003 are available at http://zebrafish.mgh. harvard.edu/mapping/ssr_map_index.html. Oligonucleotide primers for PCR to identify additional polymorphisms were designed on the basis of available zebrafish genomic sequence obtained through Blastn searches of trace data from the Sanger Institute (http://trace.ensembl.org/perl/ ssahaview? server=danio_rerio) using zebrafish plcg1 coding sequence. The oligonucleotide sequences are as follows: 2182, 5'-GTTATGGC TAAATTGAGACTCA-3'; 2185, 5'-CTTACACTCGATGCATCTGC-3'; $2115,5^{\prime}$-GATGACAATGTAGACATGCAAT-3'; and $2116,5{ }^{\prime}$-GTCT GCTCGGGTGGACTTTAA-3'. The sequence of the plcg1 intron 1-exon 1 junction was obtained using the Universal Genomewalker kit according to manufacturer's instructions (BD Biosciences Clontech) with wildtype and mutant genomic DNA as starting material and the following oligonucleotides: 5'-TAGAGGATGACGAAGCAGTGGGCCTGGT-3' and 5'-GGCCGGGTCGTGACGTCCTCCACATAT-3' for PCR. Resultant PCR fragments were sequenced directly. Polymorphism analyses and sequence comparisons were performed using SeqMan alignment software (DNASTAR, Inc.).

\section{Injections}

Morpholinos (Gene Tools) and mRNA were injected into embryos as described elsewhere (Lawson et al. 2002). Sequence for the plcg1 Morpholino is 5'-ATTAGCATAGGGAACTTACTTTCG-3'. Genomic DNA from injected embryos was isolated as described elsewhere (Roman et al. 2002) and genotyped using oligonucleotide primers 2182 and 2185.

\section{Imaging}

Imaging of blood vessels in TG(fli1:egfp $)^{y 1}$ embryos was performed using a multiphoton laser or confocal laser microscope as described previously (Lawson and Weinstein 2002a). Transmitted light images were obtained with a Leica MZ12 or Zeiss Axiophot2 microscope equipped with a ProgRes $\mathrm{mF}$ digital camera (Jenoptik).
Cloning

Sequences encompassing the plcg13' UTR and start codon were obtained using available zebrafish EST (GenBank accession nos. AW510269, BI979386, AW281801, BM778133, AW279908, AI385081, and AI397215) and genomic trace sequence. The plcg1 start codon was obtained through a tBlastn search of zebrafish genomic traces using the rat Plcgl amino acid sequence. The full-length coding sequence of zebrafish plcg1 was amplified by PCR using cDNA from wild-type embryos using the following oligonucleotides: 5'-TTACTAGTGAACAAACAGGGGAAATGGCT-3' and 5'-TTTCTAGACTGCTCGGTTTACGCTCGGTTAT-3' and TOPO-cloned into pCR2.1 (Invitrogen) to give pCRplcg1CDS. Additional plcg1 cDNA sequence was obtained or confirmed by cycle sequencing of reverse transcriptase PCR (RT-PCR) products using the BigDye sequencing kit and an ABI 310 capillary sequencer according to manufacturer's instructions (Applied Biosystems). A fragment containing the full-length plcg1 coding sequence was digested from pCRplcg1CDS and cloned into pCS2+ to give pCSplcg1CDS. Subsequently, a $6 \times$ myc epitope tag was cloned in frame of the $5^{\prime}$ end of plcg1 coding sequence in pCSplcg1 CDS to give pCSMTplcg1. pCSMTplcg1 was digested with NotI and used as template for mRNA synthesis using the mMessage mMachine kit according to manufacturer's protocols (Ambion).

Whole mount in situ hybridization

Antisense mRNA probes for ephrin-B2a, notch5, and fli1 were prepared as described (Lawson et al. 2001). To derive a plcg1 riboprobe, pCRplcg1CDS was linearized with HindIII and transcribed using T7 polymerase. Whole-mount in situ hybridization was performed as described elsewhere (Hauptmann and Gerster 1994).

GenBank accession numbers

Zebrafish plcg1 coding sequence, AY163168; plcg1 intron/exon containing CA repeat 2182-2185, AY163169; zebrafish plcg1 intron 1-exon 2 boundary, AY163170.

\section{Acknowledgments}

We thank the members of the "screen team": Michael Tsang, Neil Hukriede, Susan Lyons, Milton English, and Lin Li. We are grateful to Beth Roman, who provided advice on mapping. We thank the Wellcome Trust and Sanger Institute for making zebrafish genomic trace sequence readily available. We also thank Van Pham for helpful technical assistance and the members of the NIH zebrafish facility staff.

The publication costs of this article were defrayed in part by payment of page charges. This article must therefore be hereby marked "advertisement" in accordance with 18 USC section 1734 solely to indicate this fact.

\section{References}

Brown, L.A., Rodaway, A.R., Schilling, T.F., Jowett, T., Ingham, P.W., Patient, R.K., and Sharrocks, A.D. 2000. Insights into early vasculogenesis revealed by expression of the ETS - domain transcription factor Fli-1 in wild-type and mutant zebrafish embryos. Mech. Dev. 90: $237-252$

Carmeliet, P., Ferreira, V., Breier, G., Pollefeyt, S., Kieckens, L., Gertsenstein, M., Fahrig, M., Vandenhoeck, A., Harpal, K., Eberhardt, C., et al. 1996. Abnormal blood vessel development and lethality in embryos lacking a single VEGF allele. Nature 380: 435-439.

Draper, B.W., Morcos, P.A., and Kimmel, C.B. 2001. Inhibition of zebrafish fgf8 pre-mRNA splicing with morpholino oligos: A quantifiable method for gene knockdown. Genesis 30: 154-156.

Falasca, M., Logan, S.K., Lehto, V.P., Baccante, G., Lemmon, M.A., and Schlessinger, J. 1998. Activation of phospholipase C gamma by PI 3-kinase-induced $\mathrm{PH}$ domain-mediated membrane targeting. EMBO J. 17: 414-422.

Hauptmann, G. and Gerster, T. 1994. Two-color whole-mount in situ hybridization to vertebrate and Drosophila embryos. Trends Genet. 10: 266.

Isogai, S., Horiguchi, M., and Weinstein, B.M. 2001. The vascular anatomy of the developing zebrafish: An atlas of embryonic and early larval development. Dev. Biol. 230: 278-301. 
Lawson, N. and Weinstein, B. 2002a. In vivo imaging of embryonic vascular development using transgenic zebrafish. Dev. Biol. 248: 307.

. 2002b. Arteries and veins: Making a difference with zebrafish. Nat. Rev. Genet. 3: 674-682.

Lawson, N.D., Scheer, N., Pham, V.N., Kim, C.H., Chitnis, A.B., Campos-Ortega, J.A., and Weinstein, B.M. 2001. Notch signaling is required for arterial-venous differentiation during embryonic vascular development. Development 128: 3675-3683.

Lawson, N.D., Vogel, A.M., and Weinstein, B.M. 2002. sonic hedgehog and vascular endothelial growth factor act upstream of the Notch pathway during arterial endothelial differentiation. Dev. Cell 3: 127136.

Liang, D., Chang, J.R., Chin, A.J., Smith, A., Kelly, C., Weinberg, E.S., and $\mathrm{Ge}, \mathrm{R}$. 2001. The role of vascular endothelial growth factor (VEGF) in vasculogenesis, angiogenesis, and hematopoiesis in zebrafish development. Mech. Dev. 108: 29-43.

Liao, H.J., Kume, T., McKay, C., Xu, M.J., Ihle, J.N., and Carpenter, G. 2002. Absence of erythrogenesis and vasculogenesis in Plcg1-deficient mice. J. Biol. Chem. 277: 9335-9341.

Mukouyama, Y., Shin, D., Britsch, S., Taniguchi, M., and Anderson, D.J. 2002. Sensory nerves determine the pattern of arterial differentiation and blood vessel branching in the skin. Cell 109: 693-705.

Nasevicius, A., Larson, J., and Ekker, S.C. 2000. Distinct requirements for zebrafish angiogenesis revealed by a VEGF-A morphant. Yeast 17: 294-301.

Parsons, M.J., Pollard, S.M., Saude, L., Feldman, B., Coutinho, P., Hirst, E.M., and Stemple, D.L. 2002. Zebrafish mutants identify an essential role for laminins in notochord formation. Development 129: $3137-$ 3146.

Rhee, S.G. 2001. Regulation of phosphoinositide-specific phospholipase C. Annu. Rev. Biochem. 70: 281-312.

Roman, B.L., Pham, V.N., Lawson, N.D., Kulik, M., Childs, S., Lekven, A.C., Garrity, D.M., Moon, R.T., Fishman, M.C., Lechleider, R.J., et al. 2002. Disruption of acvrll increases endothelial cell number in zebrafish cranial vessels. Development 129: 3009-3019.

Stalmans, I., Ng, Y.S., Rohan, R., Fruttiger, M., Bouche, A., Yuce, A., Fujisawa, H., Hermans, B., Shani, M., Jansen, S., et al. 2002. Arteriolar and venular patterning in retinas of mice selectively expressing VEGF isoforms. J. Clin. Invest. 109: 327-336.

Takahashi, T. and Shibuya, M. 1997. The $230 \mathrm{kDa}$ mature form of KDR/ Flk-1 (VEGF receptor-2) activates the PLC-gamma pathway and partially induces mitotic signals in NIH3T3 fibroblasts. Oncogene 14: 2079-2089.

Takahashi, T., Yamaguchi, S., Chida, K., and Shibuya, M. 2001. A single autophosphorylation site on KDR/Flk-1 is essential for VEGF-A- dependent activation of PLC-gamma and DNA synthesis in vascular endothelial cells. $E M B O$ J. 20: 2768-2778.

Thompson, M.A., Ransom, D.G., Pratt, S.J., MacLennan, H., Kieran, M.W., Detrich III, H.W., Vail, B., Huber, T.L., Paw, B., Brownlie, A.J., et al. 1998. The cloche and spadetail genes differentially affect hematopoiesis and vasculogenesis. Dev. Biol. 197: 248-269.

Visconti, R.P., Richardson, C.D., and Sato, T.N. 2002. Orchestration of angiogenesis and arteriovenous contribution by angiopoietins and vascular endothelial growth factor (VEGF). Proc. Natl. Acad. Sci. 99: 8219-8224.

Westerfield, M. 1993. The zebrafish book. University of Oregon Press, Eugene, OR.

Wilde, J.I. and Watson, S.P. 2001. Regulation of phospholipase C gamma isoforms in haematopoietic cells: Why one, not the other? Cell Signal 13: 691-701.

Zachary, I. and Gliki, G. 2001. Signaling transduction mechanisms mediating biological actions of the vascular endothelial growth factor family. Cardiovasc. Res. 49: 568-581. 


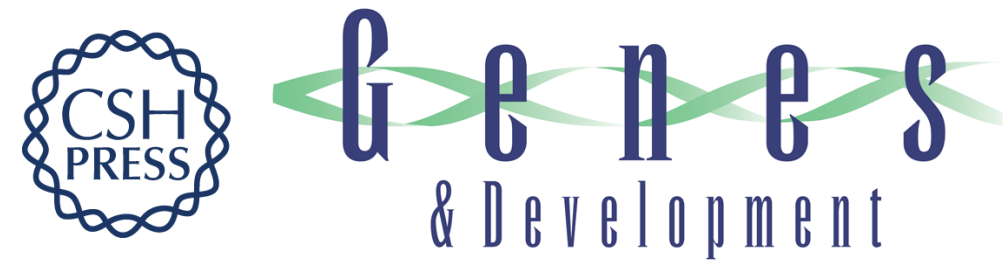

\section{phospholipase $C$ gamma-1 is required downstream of vascular endothelial growth factor during arterial development}

Nathan D. Lawson, Joshua W. Mugford, Brigid A. Diamond, et al.

Genes Dev. 2003, 17:

Access the most recent version at doi:10.1101/gad.1072203

References This article cites 23 articles, 6 of which can be accessed free at:

http://genesdev.cshlp.org/content/17/11/1346.full.html\#ref-list-1

License

Email Alerting

Receive free email alerts when new articles cite this article - sign up in the box at the top

Service right corner of the article or click here.

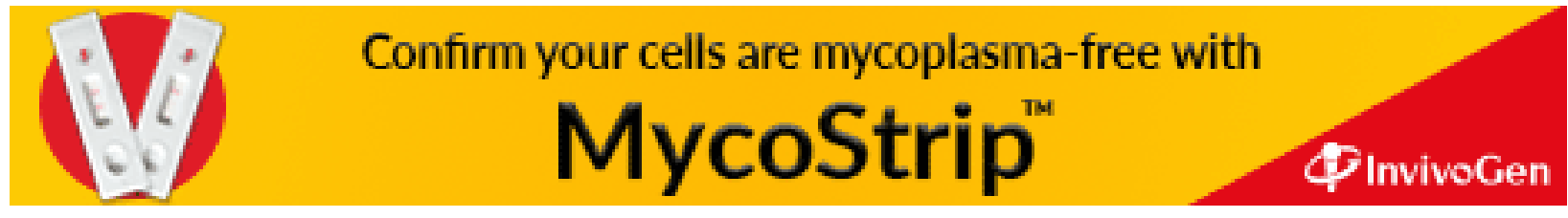

